\title{
MAROKKO IN DER NACHKRIEGSZEIT
}

\author{
Von Karl Suter \\ Mit 5 Abbildungen
}

\section{BEVOLKERUNG UND STÄDTE1}

Auch Marokko hat die Kriegsjahre stark gespürt und leidet heute noch sehr unter ihren Nachwehen. Die Lebenskosten sind hoch, die Löhne verhältnismäßig niedrig und wichtige Nahrungsmittel, wie zum Beispiel Brot, rationiert. Für den Ankauf von Rohstoffen, Fabrikaten und landwistschaftlichen Erzeugnissen im Ausland fehlen zum guten Teil die Devisen. Der Überseeverkehr stockt wegen Mangels an Schiffsraum. Es braucht Wochen, um einen Schiffs- oder Flugplatz nach Europa zu ergattern. Auch im Innern sind die Transportmöglichkeiten noch ungenügend. Der Wagenpark, der vor dem Kriege aus 68000 Autos bestand, ist stark verbraucht. Nur wenige Europäer besitzen noch einen eigenen Wagen. Das wiegt schwer in einem Land, wo die Entfernungen groß sind und sich der Verkehr hauptsächlich auf der Straße und nicht auf der Schiene abwickelt. Die Postkurse sind überlastet und unzureichend. Aber das Land ist zum Glück nicht verwüstet. Mit neuem Schwung hat die wirtschaftliche Entwicklung wieder eingesetzt. In der Landwirtschaft werden die größten Anstrengungen gemacht, um die Ackerflur viel stärker als bis anhin zu nutzen, und im Bergbau untersucht und erschließt man neue, weite Gebiete. Die Zahl der industriellen Betriebe nimmt zu, und Städte und Häfen wachsen. Diese wirtschaftliche Wiederbelebung erfolgt unter dem harten Druck einer ständigen Zunahme der Bevölkerung. Es gilt, dieser Arbeit und Brot zu verschaffen. Man schätzt gegenwärtig die Zahl der Einwohner von Französisch-Marokko auf Grund der Rationierungsausweise - genaue Zählungen gibt es immer noch nicht, da für die Eingeborenen keine Zivilstandsregister geführt werden - auf 8,4 Millionen; im Jahre 1921 waren es bloß 3,5 Millionen und 1936 6,2 Millionen. Die starke Zunahme geht auf den Einzug von Ordnung, Ruhe und Sicherheit unter den Eingeborenenstämmen, die sich vor der Errichtung des französischen Protektorats (1912) oft äußerst blutig befehdeten, und auf die erfolgreiche Bekämpfung verschiedener Seuchen zurück. Der mohammedanische Teil der Bevölkerung, der sich aus Arabern und Berbern zusammensetzt, macht gegenwärtig knapp $94 \%$ aus; auf die Juden entfallen gut $2 \%$ (1943 196000) und auf die Europäer $4 \%$ (1943 339000). Auch die Zahl der Europäer, die 1936 bloß 203000 betrug, hat stark zugenommen, vor allem durch Einwanderung während der Kriegszeit. Die weiße Bevölkerung, die zu $85 \%$ aus Franzosen und zu fast $15 \%$ aus Spaniern und Italienern besteht, wohnt zum größten Teil (70 \%) in den Städten, hauptsächlich in Casablanca. Hier ist ihre Zahl von 72000 des Jahres 1936 auf gegenwärtig rund 150000 angestiegen. Überhaupt zeigt "Casa» ein erstaunliches Wachstum. Seine gesamte Bevölkerung schnellte im gleichen Zeitraum von 257000 auf rund 600000 empor. Noch im Jahre 1907, beim ersten französischen Einmarsch, war Casa eine kleine, unansehnliche Eingeborenenstadt mit einigen wenigen tausend Einwohnern. Doch das änderte sich rasch dank der Tatkraft und des Weitblickes des ersten Generalresidenten von Marokko, Marschalls Lyautey. Er hatte beschlossen, an dieser Stelle der $750 \mathrm{~km}$ langen, buchtenarmen und wenig zugänglichen Küste den großen Hafen des Landes zu errichten. Zwei

1 Eindrücke von einer im Juli und August 1946 ausgeführten Reise. Die meisten Angaben wurden in Gesprächen mit leitenden Personen aus der Landwirtschaft, dem Bergbau und der Industrie gewonnen; einige Hinweise stammen aus dem "Bulletin économique et social du Maroc", das seit 1945 wieder erscheint. 


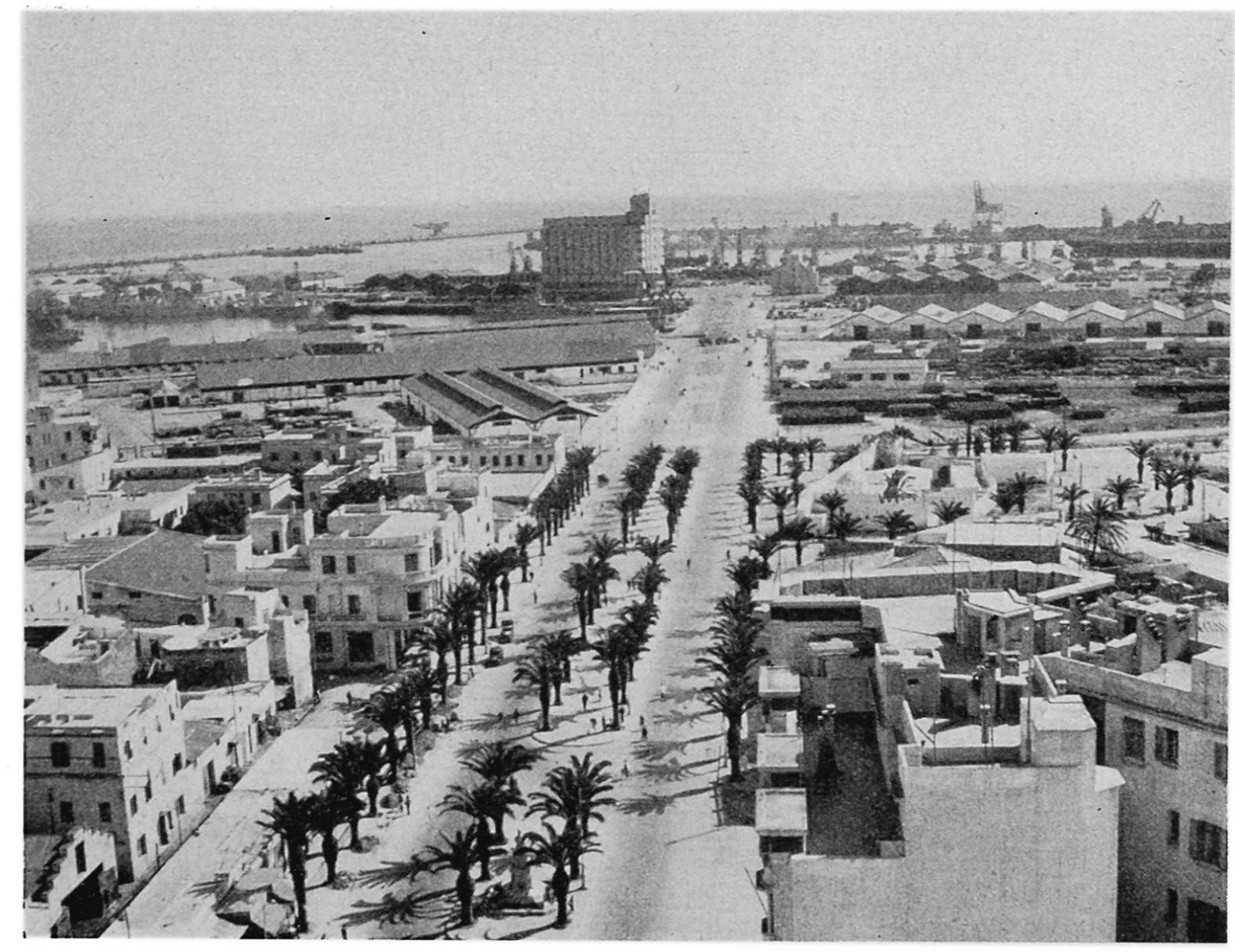

Fig. 1. Hafen von Casablanca mit Zufahrtstraße (von der Place de la France kommend)

gewaltige Molen, eine äußere von 2,5 km und eine Quermole von 1,6 km Länge, wurden in das Meer vorgetrieben. Sie schützen zusammen eine Wasserfläche von 140 ha und 9-11 m Tiefe, in die die größten Schiffe einfahren können. Der Hafen ist mit allen modernen Einrichtungen ausgestattet, so mit einem Dock, mit Silos, die zusammen 300000 Tonnen Getreide zu fassen vermögen, und mit zahlreichen Lagerschuppen. Casa bewältigt vier Fünftel der marokkanischen Ein- und Ausfuhr. Es besitzt ein reiches Hinterland, mit dem es ausgezeichnete Straßen und Bahnen verbinden, so mit Meknes und Fes im Norden und mit Marrakesch im Süden.

An die alte Eingeborenenstadt, die Medina, die sich längs des Hafens ausdehnt, reihten sich rasch neue Quartiere an. Im Süden entstand die "nouvelle médina ", ein volksreiches, großes Araberviertel, das sich aber bereits wieder als zu klein erweist. Im Westen dehnt sich die Europäerstadt, die "ville nouvelle " aus. Sie ist an ihren breiten, asphaltierten und von Bäumen eingerahmten Straßenzügen und großen Plätzen und an ihren gepflegten Gärten und Wohnhäusern auf den ersten Blick zu erkennen. Immer sind in Marokko, wie es das Gesetz vorschreibt, Eingeborenen- und Europäerviertel voneinander streng getrennt. Das einheimische Stadtbild darf nicht durch europäische Bauten verschandelt werden. Im Osten von Casablanca, zwischen Roches Noires und der Pointe d'Oukkacha, ist ein neues, großes Quartier im Entstehen begriffen. Hier hat sich die seht junge marokkanische Industrie festgesetzt. Schon finden sich da Nahrungsmittelfabriken, Bierbrauereien und chemische Fabriken. Immer neue Scharen von Eingeborenen strömen vom Lande, dem Bled, herbei, angelockt durch die Betriebsamkeit und die Verdienstmöglichkeiten. Nun soll es auch noch das wichtigste Industriezentrum des Landes werden. Im Fabrikviertel von Roches Noires ist für die Eingeborenen der Bau einer riesigen Wohnsiedlung vorgesehen.

So steht Casa von neuem bevölkerungspolitisch und siedlungsgeographisch in einem Entwicklungsvorgang großen Ausmaßes, dessen Ende noch nicht abzusehen ist. Úberall wird hier, ist nur einmal die Materialknappheit behoben, gebaut werden. 
Die Wohnungsnot ist groß, und Hotelzimmer sind schwer zu erhalten. Man denkt daran, das Hauptverkehrsnetz vollständig umzulagern. Bereits ist der Mittelpunkt der Stadt, die Place de la France, auf Kosten der alten Medina erweitert worden. Besonders schwierig ist es, für die ständig wachsende Stadt das nötige Trinkwasser zu beschaffen. Die heutige Trinkwasserversorgung erweist sich, wenigstens im Sommer, als unzureichend. Dann fließt das Wasser, das zum Teil weit her kommt, so aus dem $130 \mathrm{~km}$ entfernten Gebiet des Fouarat im Südosten von Port-Lyautey, in Küchen und Waschräumen äußerst spärlich, und ist oft gar zu gewissen Tagesstunden gänzlich abgestellt. Im Sommer ist es in den Restaurants häufig unmöglich, vor einer Mahlzeit seine Hände zu waschen.

Einst war Marrakesch die größte Stadt des Landes. Es ist durch Casablanca auf den zweiten Platz zurückversetzt worden. Es zählt gegenwärtig ungefähr 220000 Einwohner, nämlich 13000 Europäer, 190000 Mohammedaner und 17000 Juden. Dazu kommt eine nur vorübergehend sich aufhaltende Bevölkerung, die das Bled, insbesondere die Täler und Fußregionen des Hohen Atlas, bewohnt. Sie beläuft sich, je nach Jahreszeit, auf 30000 bis 50000 Menschen. Marrakesch ist die Haupt- und Vergnügungsstadt des Südens.

Das Wohnquartier der Europäer, das "Guéliz", das sich im Westen der Medina befindet, ist eine kleine, saubere Gartenstadt von regelmäßiger Anlage und mit europäischen Wohnhäusern, Villen und Verwaltungsgebäuden. Darunter fällt das neue, in modernem arabischem Stil gebaute Tribunal de justice besonders auf. Zwischen Guéliz und Medina liegt das "quartier d'hivernage ", das dazu bestimmt ist, den fremden Besuchern Unterkunft, Erholung und Zerstreuung zu bieten. Es besteht aus Parkanlagen, einem Kasino und einigen Hotels, darunter dem Mamounia, dem ersten Hotel des Platzes, und dem Moghreb. Marrakesch ist bereits wegen seiner milden Winter und seiner landschaftlichen Schönheit ein bedeutender Fremdenort Marokkos geworden. Es ist durchaus damit zu rechnen, daß die Zahl der Gäste, die hier während des Winterhalbjahres ihre Ferien verbringen, in den nächsten Jahren noch mehr zunimmt. Die Fremden rühmen den wahrhaft bezaubernden Anblick, den dann die Stadt im Schmucke ihres Palmengürtels und ihrer Orangenhaine und mit dem nahen, schneebedeckten Hohen Atlas als Hintergrund bietet. Im Sommer dagegen gleicht die Landschaft, von wenigen, künstlich bewässerten grünen Flächen abgesehen, einer braunrot gefärbten, ausgebrannten Wüstenei. Dann herrscht wochenlang große Hitze mit Schattentemperaturen von $45-49^{\circ} \mathrm{C}$. Sicherlich wird Marrakesch in naher Zukunft an Bedeutung noch zunehmen. Es ist geplant, weite, wenig fruchtbare Flächen künstlich zu bewässern und in Obst- und Ackerland umzupflügen und ferner das Atlasgebirge noch in stärkerem $\mathrm{Maße}$ touristisch und sportlich zu erschließen.

Auch andere marokkanische Städte haben große Bevölkerungszunahmen zu verzeichnen, wenn auch nicht so wie Casablanca. Besonders stark war der Zuwachs in jenen Orten, wo sich eine größere Zahl von Europäern befindet, die als Unternehmer und Kaufleute den Eingeborenen leicht Arbeit zu bieten vermögen, wie in Rabat, der Landeshauptstadt, in Meknes, in Fedala, das sich zum wichtigsten Petrolimporthafen Marokkos und einem bedeutenden Fischereiplatz entwickelt hat, oder in Port-Lyautey und Safi. Port-Lyautey wird, wie es scheint, der wichtigste Hafen des nördlichen Teils von Marokko werden. Es besitzt in den Regionen des Rharb, von Meknes und Fes ein reiches Hinterland, das dieser Stadt einen großen Teil seiner Ackerbauprodukte zur Ausfuhr liefert. Die gleiche Rolle ist für Südmarokko dem Hafen von Safi zugedacht, der dank der Ausfuhr von Phosphaten aus dem Gebiete von Louis-Gentil zum zweitwichtigsten Hafen des Landes aufgerückt ist. Marokko zählt heute 19 Städte mit mehr als 10000, darunter fünf mit sogar über 100000 Einwohnern, nämlich Casablanca, Rabat, Fes, Meknes und Marrakesch.

\section{ANBAU ;UND AGRARREFORM}

Die landwirtschaftlich nutzbare Fläche Marokkos wird auf 15 Millionen Hektaren geschätzt. Das ist nicht gerade viel im Vergleich zur Größe des ganzen Landes, die ungefähr 40 Millionen Hektaren beträgt. Weitaus an vorderster Stelle steht der Getreidebau, dem mehr als $90 \%$ der genutzten Fläche zugeteilt sind. Er bildet die Grundlage 
für die Ernährung der Bevölkerung. Ihm liegt die große Zahl der Eingeborenen ob, die als Ackerbauer und Hirten im Bled leben. Sie pflanzen im Winter Weizen und Gerste, im Sommer Mais an.

Noch immer wickelt sich der Anbau in uralten, rückständigen Formen ab. Die Hektarerträge sind deshalb nicht hoch. Sie machen im Durchschnitt für Weizen 4-5 q, für Gerste 6-8 q und für Mais 3-7 q aus. Die Ernteerträge schwanken aber von Jahr zu Jahr je nach den Witterungsumständen. Das zeigen die folgenden Zahlen für die gesamte Getreideproduktion: 193716 Millionen Doppelzentner, 193937 Millionen, 194138 Millionen, 194418 Millionen. Aus den letzten 12 Jahren ergibt sich ein jährlicher Durchschnitt von 23 Millionen Doppelzentner, der gerade groß genug ist, um den einheimischen Bedarf zu decken. Doch häufig, ja fast jedes zweite Jahr, bleiben die Erträge unter diesem Mittel. Marokko sieht sich dann gezwungen, Getreide einzuführen. Das wäre nicht nötig, wenn es die Einrichtungen besäße, um den Getreideüberschuß der guten Jahre für die Notzeiten aufzustapeln. Statt dessen gibt es den Überschuß ans Ausland ab und erweckt damit den unzutreffenden Eindruck, es sei ein bedeutender Getreidelieferant. Bleiben die Niederschläge, wie das im Jahre 1945 der Fall war, während der Wachstumsperiode im Winterhalbjahr fast ganz aus, was glücklicherweise selten vorkommt, so muß notgedrungen eine Fehlernte eintreten. Damals betrug die Gesamtproduktion nur 4 Millionen Doppelzentner. Die Ernährungslage war katastrophal, und das Ausland mußte mit bedeutenden Zuschüssen aushelfen.

Rückständig wie der Landbau ist auch noch die Art, wie die bäuerliche Arbeit entlöhnt wird. Meist ist noch das System des "khammessat" in allen seinen Abarten üblich. Wer das Feld seines Herrn bestellt, bekommt für seine Arbeit den fünften Teil des Kornertrages. Diese Art der Arbeitsentschädigung ist bei den mengenmäßig von Jahr zu Jahr sehr schwankenden Erträgen unbefriedigend. Sie lastet schwer auf dem Bauernstand und hindert jeden sozialen Fortschritt. Doch die Anzeichen, vom Fünftel abzurücken, mehren sich. Die Eingeborenen erkennen allmählich die Vorzüge einer regelmäßigen und in Bargeld stattfindenden Entlöhnung, wie sie der europäische Kolonist durchführt. Ein feststehender Taglohn, auf den sie bestimmt zählen können, würde ihnen das Anschaffen von Nahrungsmitteln, Kleidern und anderen Dingen stark erleichtern.

Nicht ganz so machtlos wie der Eingeborene steht der Europäer der Natur gegenüber. Er vermag den Härten des Klimas dank seinen viel besseren Anbaumethoden bis zu einem gewissen Grade zu begegnen. Er weiß, daß man in Marokko oft schnell arbeiten muß, um zum Beispiel von den Regen zu profitieren oder um eine vom «chergui», dem trockenen Südwind, bedrohte Ernte einzubringen. Das ist aber bloß mit Hilfe der modernsten landwirtschaftlichen Maschinen möglich. Die Erträge des Europäers sind deshalb weit größer und weniger schwankend. Er erhält im Mittel pro Hektare 8-12 q Weizen und 9-13 q Gerste. Indessen nimmt die Kultur von Getreide beim Europäer, im Gegensatz zum Eingeborenen, keine überragende Stellung ein; er pflanzt nur kleine Flächen damit an.

Die Zahl der europäischen Bauern ist nicht sehr groß; sie beträgt ungefähr 4000 . Davon sind 3500 Franzosen mit zusammen 30000 Angehörigen. Die europäischen Einwanderer haben sich in ganz bestimmten Gebieten, die sich durch ein günstigeres Klima und gute Böden auszeichnen, angesiedelt, so an der Küste, besonders um Casablanca, Rabat und Port-Lyautey, und ferner im Innern um Meknes. Ihre Höfe sind in der Regel $50-300$ ha groß. Kleinere Güter von $10-20$ ha trifft man vor allem um die größeren Städte; sie bringen fast ausschließlich Gemüse hervor. Ein Teil davon wird als Frühgemüse nach Europa verschickt. Die europäische Kolonisation hatte in erster Linie den Zweck, die Eingeborenenbevölkerung wirtschaftlich zu heben und die Agrarproduktion Marokkos zu steigern.

Das ist bis heute, wie die folgenden Zahlen zeigen, zu einem schönen Teil gelungen. Im Jahre 1928 betrug zum Beispiel bei den Europäern die mit Südfrüchten bestellte Fläche nur rund 700 ha, im Jahre 1939 dagegen schon 5500 ha und 1946 gar 15500 ha. Das macht heute mit den Kulturen der Eingeborenen zusammen 18000 ha aus. Es werden namentlich Orangen gehalten, deren Produktion gewaltig zugenommen hat. 1920 führte Marokko nur $212 \mathrm{~kg}$ Orangen aus, 1930 dagegen $3000 \mathrm{q}$ und $194012000 \mathrm{t}$. Stark ist auch der Anbau von Frühgemüse ausgedehnt worden, nämlich von 20000 ha des Jahres 1939 auf 35000 ha des Jahres 1946, und ferner der von Tabak (1930 19 ha mit einer Produktion von $23 \mathrm{t}, 1944856$ ha mit einer solchen von $1005 \mathrm{t}$ ) und Baumwolle. 


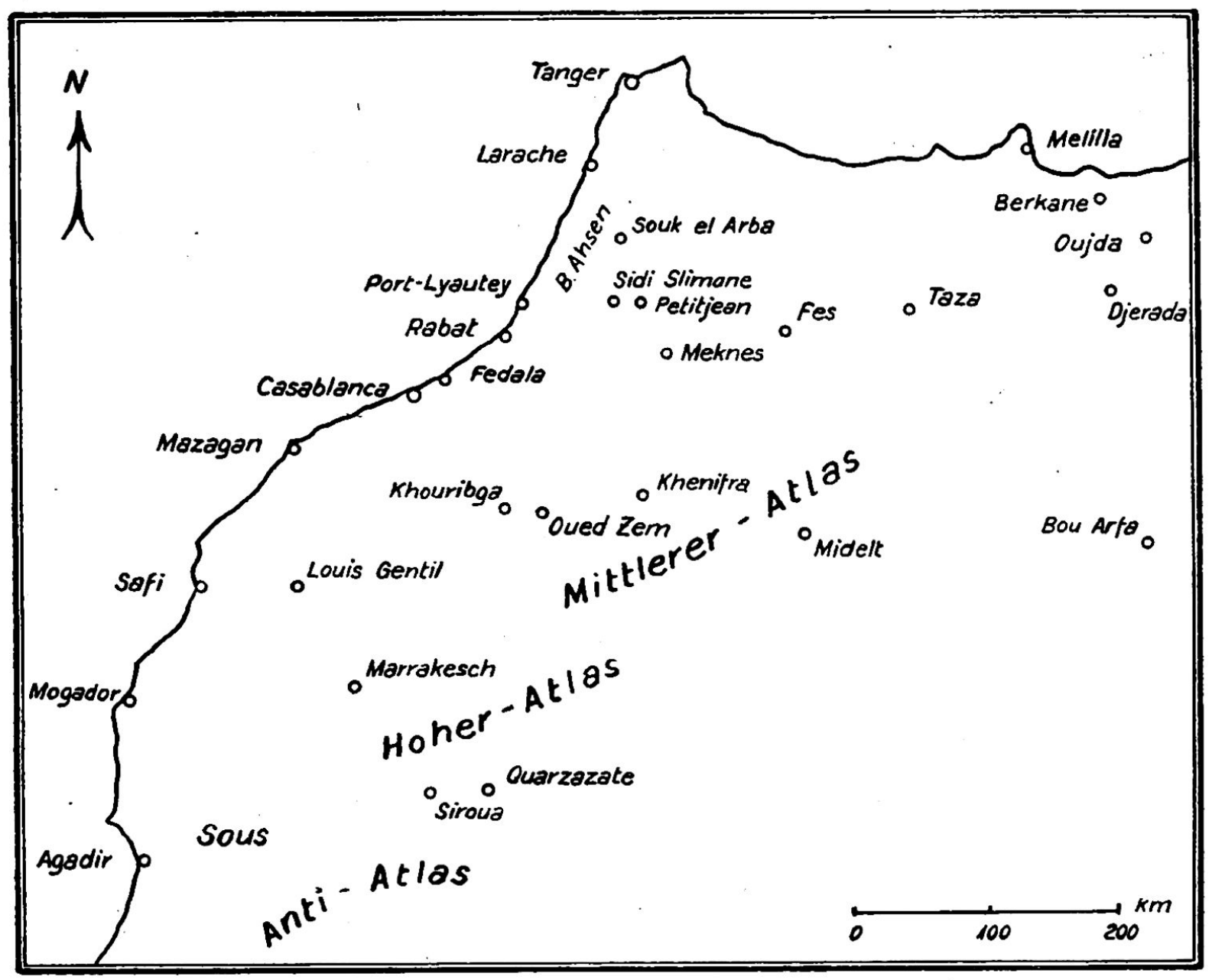

Fig. 2. Übersichtsskizze von Marokko

Es scheint augenblicklich, daß die Baumwolle in Marokko eine gewisse Zukunft hat. Dagegen hat die Rebe an Bedeutung viel verloren; ging doch ihre Anbaufläche von 772000 ha des Jahres 1938 auf 230000 ha des Jahres 1945 zurück. An diesem Rückgang trägt die Reblaus die Hauptschuld. Der Kampf gegen diesen Schmarotzer muß mit allen Mitteln aufgenommen werden, soll es nicht um den marokkanischen Rebbau geschehen sein. Die Rebgebiete bei Casablanca, Meknes und Fes müssen von Grund auf umgestaltet werden.

Das Protektorat wird auf einen weitern größeren Zuzug von geschulten und erfahrenen europäischen Kolonisten, die den Eingeborenen als Pioniere eines neuzeitlichen Landbaus voranzugehen haben, angewiesen sein. Die bereits ansässigen weißen Siedler können bei fortschreitender Kultivierung des Landes diese Aufgabe unmöglich allein lösen; denn sie sind hiefür an Zahl viel zu klein. Aber auch in Zukunft soll am bewährten Grundsatz, den Eingeborenen nicht durch den europäischen Einwanderer zu verdrängen, festgehalten werden.

Die Franzosen haben in den drei Jahrzehnten, die seit der Besetzung Marokkos verstrichen sind, ein beachtenswertes kolonisatorisches Werk vollbracht. Sie haben viele einst trostlos öde und trockene, aber auch versumpfte Gebiete anbaufähig gemacht, so zum Beispiel die Ebene von Beni-Ahsen.

Das Wasser ihres Abflusses, des Oued Beth, wurde in den Jahren 1931-34 bei El Kansera, $35 \mathrm{~km}$ südlich von Sidi Slimane, durch eine $175 \mathrm{~m}$ lange und $43 \mathrm{~m}$ hohe Mauer aufgestaut. Es entstand so ein See von einem Fassungsvermögen von 225 Millionen Kubikmeter, der eine Bewässerung von ungefähr 30000 ha gewährleistet. Bis Ende 1945 waren davon aber erst 6000 ha genutzt. Ein großartiges 
Netz von Kanälen und Gräben breitet sich über die Ebene aus und leitet das Wasser zu den zahlreichen Gütern. Der Hauptkanal allein mißt $47 \mathrm{~km}$. Wohin der Blick auch immer geht, umfängt er Getreidefelder, die aber nur in trockenen Jahren bewässert werden, und reich benetzte Gemüse-, Tabak- und Obstkulturen. Die Agrumen bedecken heute allein schon eine Fläche von 2600 ha. Damit sind die Anbaumöglichkeiten dieses Gebietes jedoch keineswegs etwa erschöpft. Im Jahre 1946 hat man hier, angespornt durch die geglückten Versuche in der Landschaft Berkane in Nordostmarokko, mit dem Anbau von Niora begonnen, eines aus Südamerika stammenden, an verschiedenen Vitaminen reichen Gewürzes, das man den Konserven mit Vorteil beigibt. Die Niora, mit der man die freibleibenden Nutzstreifen zwischen den Orangenbäumen bestellt, gedeiht hier bestenś und liefert hohe Erträge. Für die Zukunft ist auch das Anpflanzen anderer Gewächse geplant; nur müssen vorher noch verschiedenartige Pflanzenschädlinge systematisch bekämpft werden. Die schweizerischen Produkte für Schädlingsbekämpfung, insbesondere jene der Firma Geigy in Basel, erfreuen sich eines zusehends wachsenden Zuspruchs.

In dieser stark genutzten Bewässerungslandschaft, dem "bled seguia ", herrschen die Güter von mittlerer und kleiner Betriebsgröße vor. Eine Fläche von 20-50 ha wird für den europäischen Kolonisten als hinreichend angesehen. Ist das Land nicht bewässerbar, ist es also "bled bour", so müssen ihm mindestens 100-125 ha zur Verfügung stehen. Ein Gesetz schreibt den Europäern und Eingeborenen die Mindestgröße, die die Güter in Sidi Slimane haben müssen, vor. Es regelt auch weitgehend den Kauf und Verkauf von Land und verbietet zum Beispiel dem Europäer, Grundstücke, die den Fellachen gehören, zu erwerben; das Besitztum der Eingeborenen soll voll erhalten bleiben.

Das Gebiet von Sidi Slimane weist seit 1934 eine starke Bevölkerungszunahme auf. Es wohnen hier heute 58000 Menschen gegenüber bloß ungefähr 25000 im Jahre 1916. Das Dorf selber ist eine vollständige Neugründung, die gegenwärtig bereits 700 Europäer und gegen 4000 Eingeborene zählt und mit fortschreitender Urbarmachung der Ebene an Einwohnern noch beträchtlich zunehmen wird. Allerdings gilt es vorher noch, der Malaria Herr zu werden, die fatalerweise mit der Umwandlung des einst von Sümpfen durchsetzten Bled zur Kulturoase, wohl im Zusammenhang mit dem Bau der Bewässerungsanlage, sich weiter ausgebreitet hat. Ist diese Geißel der Gegend einmal gründlich bekämpft und das lebensspendende Wasser des Oued Beth überall hin verteilt, so steht einer noch stärkeren Besiedlung nichts mehr entgegen. Die Ebene von Beni-Ahsen kann dann leicht mehr als 10000 Europäern und 100000 Eingeborenen Arbeit und Verdienst geben.

In Marokko besteht die schwerwiegende Tatsache, daß die durch den Fellachen angebaute Erde viel zu wenig abwirft. Der Boden vermag die auf ihm lebende Bevölkerung oft kaum zu ernähren. Marokko sieht sich deshalb vor die unerbittliche Notwendigkeit gestellt, seine landwirtschaftliche Produktion wesentlich zu steigern. Daß dies möglich ist, das haben die europäischen Kolonisten bewiesen. Der Eingeborene muß sich bessere Anbaumethoden aneignen und sich mit der Verwendung landwirtschaftlicher Maschinen vertraut machen. Das allein genügt aber bei weitem nicht. Fast noch wichtiger als die Mechanisierung und Motorisierung der Landwirtschaft erscheint es, überhaupt einen neuen Bauerntypus zu schaffen. Man will beim Eingeborenen Sinn und Verständnis für einen verbesserten Ackerbau wecken und so ein gewisses Gegengewicht zu seiner tief eingewurzelten fatalistischen Einstellung erzeugen.

Zur Verwirklichung dieser tiefgreifenden Reform ist im Jahre 1945 der "Secteur de modernisation du paysannat» oder abgekürzt SMP. gegründet worden. Diese "Abteilung für neuzeitlichen Landbau», die unter der Autorität des Generalresidenten und des Großveziers steht, wird an zuständiger Stelle von einem Oberen Rat für Landbau, dem Franzosen und Eingeborene angehören, geleitet. Daneben besteht als ausführendes Organ ein ständiges Sekretariat. Dieses Amt will nun aus Brach- und Weideland eine Anzahl Kollektivbetriebe in den verschiedensten Gegenden, so im Rharb, im Mittleren Atlas, in der Umgebung von Rabat und Casablanca, schaffen. Unter der Abkürzung SMP. sind auch diese Betriebe selber, die für Marokko in rechtlicher, verwaltungstechnischer und sozialer Hinsicht eine vollständige Neuschöpfung darstellen, zu verstehen.

Ein SMP. ist fürs erste ein bestimmt begrenztes Stück Land, das durch eine Gruppe von Leuten gemeinsam bewirtschaftet wird. Seine Größe richtet sich weitgehend nach seinen Anbaumöglichkeiten und hängt deshalb wesentlich von der Fruchtbarkeit seines Bodens und seinen Wasserverhältnissen ab. Ein Betrieb, der aus «bled bour» besteht, muß viel größer als ein solcher aus "bled seguia» sein. In jedem Fall aber ist die Ausdehnung der Güter auf einige hundert, ja sogar viele tausend Hektaren fest- 
gesetzt; im Durchschnitt werden sie 2000 ha umfassen. Es handelt sich dabei um Land, das den Eingeborenen, entweder einem Stamm oder einem Teil eines Stammes, seit altersher gehört und von ihnen bereits seit Jahrhunderten, aber eben extensiv, genutzt wird. Dieses Land hat unbedingt in ihrem Besitz zu bleiben. Im Rahmen der Kollektivwirtschaft bekommt jeder Eingeborene für sich und seine Familie eine Parzelle zugeteilt, die er persönlich bestellen und die ihm einen Teil der Nahrung liefern soll. Im übrigen arbeitet er in der Gemeinschaft, zuerst als Knecht und später, je nach Neigung und Leistung, in besseren Stellen.

Dem SMP. ist im Jahre 1945 durch ein Gesetz der Charakter einer öffentlich-rechtlichen Körperschaft verliehen worden. $\mathrm{Er}_{\mathrm{r}}$ ist mit allem für den Betrieb unbedingt notwendigen Material, wie landwirtschaftlichen Geräten und Maschinen und vor allem Traktoren, ausgestattet. In radikaler Lösung, ohne irgendwelche $Z$ wischenstadien, soll der Araber die gewaltige Úberlegenheit der Maschine gegenüber seinen bisherigen primitiven Mitteln kennenlernen. Dauernd benützte Geräte werden gekauft, die andern, wie zum Beispiel Traktoren für die Urbarmachung des Bodens, nur gemietet. Der SMP. sorgt für ihren Unterhalt und nötigenfalls für ihre Wiederinstandstellung. Für den Bezug des gesamten Materials steht ihm eine besondere Stelle, die "Zentrale für landwirtschaftliche Ausrüstung", zur Verfügung. Sie unterhält auch einige Reparaturwerkstätten, die so gelegen sein müssen, daß sie von verschiedenen Betrieben aus leicht erreicht werden können.

Jedem SMP. steht ein besonderer, aus seiner Mitte gewählter Rat vor. Damit wird eigentlich nur die jahrhundertealte Überlieferung der "djemaas", der Eingeborenenräte, die zur Verwaltung des Kollektivlandes schon immer bestanden haben, in veränderter, dem Unternehmen angepaßter Form fortgesetzt. Der neue Rat hat das Anbauprogramm aufzustellen, die Verteilung der Ernte vorzunehmen, den Absatz der Produkte zu besorgen, die Entlöhnung festzusetzen usw. Die eigentliche Leitung des Betriebes liegt in den Händen eines erfahrenen Technikers, dem Vorarbeiter, Mechaniker und bäuerliche. Werkmeister zur Seite stehen.

Der SMP. stellt ferner eine soziale Einrichtung dar. Lehrer, Ärzte oder Krankenpfleger, Hebammen und Fürsorger, die ständig auf dem Gute leben, haben die Eingeborenen geistig, beruflich und körperlich zu fördern und im besonderen die Kinder zu unterrichten und gesund zu erhalten. Auch sind Architekten für den Bau nicht nur von einzelnen Häusern, sondern von ganzen Siedlungen vorhanden. Denn hier auf dem SMP. macht der Eingeborene eine für ihn höchst wichtige Wandlung durch, indem er Nomadentum und Zeltdorf, den für Nordafrika charakteristischen "douar", aufgibt und endgültig zur seßhaften Lebensweise übergeht. Die zu errichtenden Dörfer und Häuser müssen selbstverständlich dem Klima und den Lebensgewohnheiten ihrer Bewohner angepaßt sein.

Die Agrarreform, die der Secteur de modernisation du paysannat anstrebt, ist also überaus einschneidend. Sie erinnert gewiß zum Teil an die russischen Kolchosen, ist indessen doch in mancher Hinsicht etwas ganz Neues. Immer muß sie den besonderen Gegebenheiten des Landes angepaßt bleiben. Für die kommenden fünf bis zehn Jahre sind im ganzen 220 Betriebe mit zusammen 440000 ha Fläche vorgesehen. Jeder einzelne SMP. soll im Durchschnitt in vier Jahren voll betriebsfähig sein, indem auf ihm jährlich ungefähr 500 ha für den Anbau hergerichtet werden. Seit dem Jahre 1945 sind ihrer einige bereits in Ausführung begriffen, so bei Meknes, Beni-Mellal und Berkane. An diesem Ort handelt es sich um eine Fläche von bloß 674 ha, bei Meknes dagegen um eine solche von 3887 ha. Man wählt mit Vorliebe solches Gelände, für das die Möglichkeit künstlicher Bewässerung durch Grund- oder Flußwasser besteht. Die bereits vorhandenen Kollektivwirtschaften stellen einen verheißungsvollen Versuch dar. Man hofft, daß dieses großzügig geplante Unternehmen Marokko den landwirtschaftlichen und sozialen Fortschritt bringt und sein Aussehen grundlegend verändert.

\section{DIE BBERGBAULICHE ENTWICKLUNG}

Marokko steht noch am Anfang seiner bergbaulichen Entwicklung. Erst seit gut zwei Jahrzehnten wird der Boden systematisch auf Mineralien untersucht. Es sind bereits zahlreiche Lagerstätten an verschiedenartigsten Bodenschätzen entdeckt worden; jedoch läßt sich heute, im gesamten betrachtet, über deren Reichhaltigkeit, Abbaumöglichkeit, Produktionsvolumen und Wirtschaftlichkeit noch kein abschließendes Urteil bilden. Allerdings scheint es im Augenblick, als ob Marokko im Bergbau eine 
gewisse Bedeutung erlangen würde. Es wäre aber falsch, aus den bisherigen Untersuchungsergebnissen allzu große Hoffnungen abzuleiten. Die Fundstellen sind, von den Phosphaten abgesehen, in ihrem Ausmaße doch wohl eher begrenzt. Ganz unabgeklärt sind zurzeit jedenfalls noch die Zukunftsaussichten beim Erdöl. Wohl steht auf Grund der seit 1930 vorgenommenen Untersuchungen fest, daß Marokko erdölführende Schichten besitzt. So sind im Jahre 1940 in einer Zone, die dem Südrand des Rifbogens zwischen Larache und Fes folgt, drei kleine Olvorkommen entdeckt worden, die seither auch ausgebeutet werden. Ihrer zwei liegen in der Umgebung von Petitjean, nämlich bei Boudra und Tselfat, und eines befindet sich in Ain-Hamra bei Souk-el-Arba. Von allen drei Bohrstellen aus wird das Öl durch Pipe-lines nach Petitjean geleitet, wo es destilliert wird. Die Gesamtproduktion ist vorderhand sehr gering; sie beläuft sich bloß auf etwas mehr als $4000 \mathrm{t}$ im Jahr. Man hofft nun, daß sich zu den ersten Bohrtürmen, die sich auf marokkanischem Boden erheben, in den nächsten Jahren weitere gesellen werden. Es ist beabsichtigt, die Bohrungen, die heute auf $300-1200 \mathrm{~m}$ hinabreichen, in weit größere Tiefen, nämlich bis zu $4000 \mathrm{~m}$, vorzutragen.

Marokko besitzt auch Kohle. Nach den bisherigen Feststellungen kommt sie allerdings nur an zwei Stellen vor, bei Aoufous, südlich von Meski (1947 entdeckt), und bei Djerada, $60 \mathrm{~km}$ im Süden von Oujda (1928 entdeckt), wo sie seit einigen Jahren abgebaut wird. Die Produktion hält sich aber in bescheidenen Grenzen. Sie betrug in den Jahren 1937-1939 jährlich $100000-120000$ t, ist dann jedoch während des Krieges, als die Einfuhr dieses Rohstoffes stockte, stark gesteigert worden. So belief sie sich im Jahre 1945 auf ungefähr $200000 \mathrm{t}$ und vermochte damit den Bedarf des Landes, der auf $268000 \mathrm{t}$ geschätzt wird, zu einem guten Teil zu decken. Die marokkanische Kohle ist ein wenig fetter Anthrazit, der sich wohl für den Hausbrand, oft aber nicht für industrielle Zwecke eignet.

An zahlreichen Stellen Marokkos kommen Erze verschiedener Art vor: Eisen, Mangan, Kupfer, Blei, Zink, Kobalt, Antimon, Zinn, Molybdän. Es ist jedoch noch ganz ungewiß, welche Bedeutung sie für die Wirtschaft des Landes erlangen werden. Sie werden erst zum allergeringsten Teil abgebaut. Noch sind die Voraussetzungen für eine Ausbeutung großen Stiles nicht geschaffen. Viele Lager finden sich in so abgelegenen Gebieten, $\mathrm{da} \beta$ dorthin überhaupt zuerst Straßen gebaut werden müssen. Dann erst können die Bergwerke erstellt werden. Ebenso wichtig ist es aber, daß im Bergbau erfahrene Techniker und Spezialisten ins Land kommen, um die für Marokko sich eignenden Abbaumethoden zu studieren und den Eingeborenen zum Bergarbeiter heranzubilden. Große, noch nicht in Ausbeutung begriffene Eisenerzlager finden sich im Sous, im Hohen und Mittleren Atlas und im Djebilet; als wichtigstes wird dasjenige von Khenifra angesehen, das mindestens 50 Millionen Tonnen Erz enthalten soll.

Marokko ist sehr reich an Manganerz, namentlich im Gebiete des Siroua-Massivs $z w i s c h e n$ dem Sous und dem Hohen Atlas. Man rechnet damit, daß allein schon die sich hier befindenden Lager, ist nur einmal der Abbau in die Wege geleitet, eine jährliche Gesamtproduktion von 250000 t ergeben werden. Dazu kommt das Lager von Bouarfa in Ostmarokko, das seit einigen Jahren abgebaut wird. Seine Erze gelangen mit der Bahn nach dem Hafen von Nemours.

Marokko besitzt überaus reiche Phosphatlager. Diese werden seit 1921 ausgebeutet. Im Jahre 1930 erreichte die Produktion ihren Höchstwert, nämlich $2098000 \mathrm{t}$, und im Jahre 1939, nachdem sie in der Zwischenzeit infolge der Weltwirtschaftskrise bedeutend zusammengeschrumpft war, 1702000 t. Marokko deckte mit dieser Menge einen beträchtlichen Teil des Weltbedarfs, der auf ungefähr 12 Millionen Tonnen geschätzt wurde. Der Krieg brachte einen neuen Rückschlag, der überaus heftig war; sank der Abbau doch im Jahre 1942 bis auf 386000 t. Seither hat sich der Absatz stark gebessert. Schon 1945 war mit 1814000 t die Vorkriegsproduktion wieder erreicht, und für 1946 ist nach den erhaltenen Auskünften der Generaldirektion des Office chérifien des 
phosphates (OCP.) in Rabat mit einer bis dahin noch nie vermerkten Ausbeutung von 2,7 Millionen Tonnen zu rechnen. Die Nachfrage nach Phosphaten ist seit Kriegsende überaus groß, und das Phosphatamt muß alles daransetzen, um ihr zu genügen. Die Schwierigkeiten liegen darin, $\mathrm{da} B$ es gegenwärtig noch an Grubenholz, an routinierten Bergarbeitern und, als Folge der katastrophalen Trockenheit des Jahres 1945, an elektrischer Kraft fehlt. Fast die gesamte ausgebeutete Menge gelangt zur Ausfuhr; denn im Lande selber ist der Verbrauch, der jährlich bloß ungefähr $30000 \mathrm{t}$ beträgt, sehr gering. Von 1921 bis 1940 waren Frankreich, Spanien und Italien die drei wichtigsten Käufer. Das hat sich durch den Krieg vollständig geändert. Seit 1943 steht England unter den Bezügern weitaus an vorderster Stelle (1945 $572000 \mathrm{t}$ ), und erst in weitem Abstande folgt Frankreich (1945 237000 t). Die Schweiz bezog 19454467 t im Werte von 270000 Schweizerfranken.

Die Phosphate sind für die Wirtschaft Marokkos von allergrößter Wichtigkeit. Schon 1939 nahmen sie unter den Exportgütern des Protektorats den ersten Platz ein.

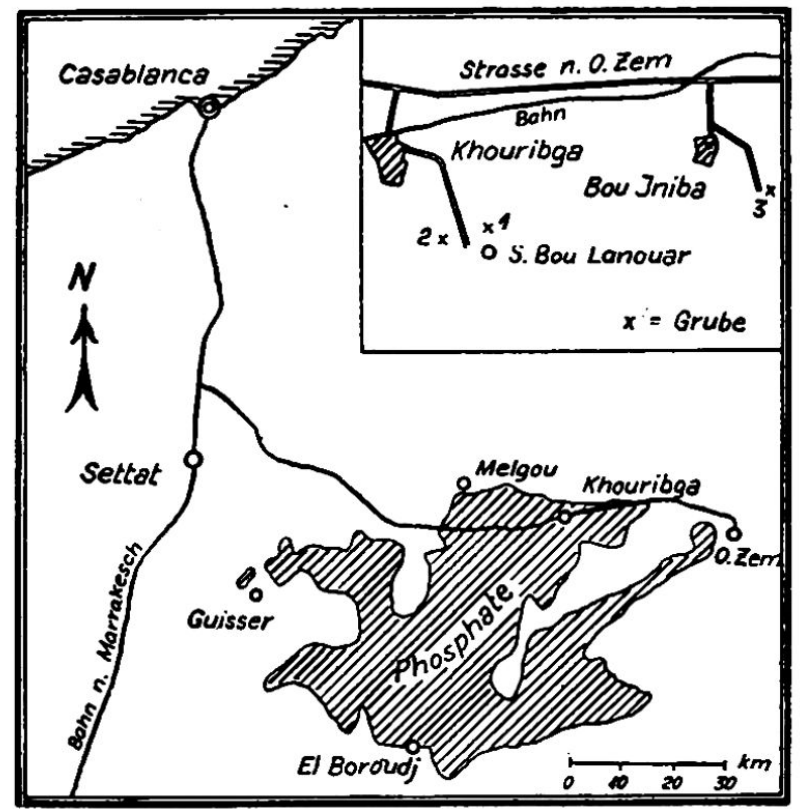

Fig. 3. Das Phosphatgebiet von Khouribga mit seinen Abbaustellen

Sie machten wertmäßig $30 \%$ und mengenmäßig gar bis $90 \%$ der gesamten Ausfuhr aus. Heute sind die Phosphate für Marokko ein besonders kostbares Ausfuhrgut, weil sie wesentlich mithelfen, ihm die nötigen Devisen zu beschaffen. Jedes Frachtschiff, das Casablanca oder Safi anläuft, kann auf sie als Rückfahrtsfracht zählen.

Die Phosphate kommen in zwei Gebieten vor, wo sie ausgedehnte Flächen bedecken. Das eine, nämlich die Hochfläche der Oulad-Abdoun, befindet sich im Norden von Marokko. Es mißt $80 \mathrm{~km}$ von Osten nach Westen, von Guisser nach Oued-Zem, und $50 \mathrm{~km}$ von Norden nach Süden, von Melgou nach El-Boroudj. In seinem Mittelpunkt liegt die Stadt Khouribga. Hier wird seit 1921 abgebaut. Das andere Lager, das von Gantour, befindet sich im Süden von Marokko, $80 \mathrm{~km}$ von Safi entfernt. Es wurde 1931 bei Louis-Gentil in Betrieb genommen.

Im Lager von Khouribga handelt es sich um mehrere phosphatführende Schichten von zusammen einigen Metern Mächtigkeit. Sie enthalten zahlreiche fossile Uberreste; so erbsengroße Koprolithen und Haifischzähne. Sie müssen also im Meere entstanden sein, und zwar, nach den geologischen $\mathrm{Be}-$ funden, zur Tertiärzeit. Zur Ausnützung taugt aber bloß eine bestimmte, im Durchschnitt 1,8 m mächtige Schicht, die zu $75 \%$ aus sehr feinen, weißlich-gelben und überaus leicht zerreibbaren Phosphatsanden besteht. Diese sind sehr feucht, weil sie 14-16\% Wasser enthalten. Das Wasser muß ihnen vor dex Verfrachtung, damit sich ihr Gewicht verringert, in besonderem, teurem Verfahren entzogen werden. Das ist nur bedingt ein Nachteil; denn die hohe Feuchtigkeit des Gesteins wirkt sich auch 
günstig aus, indem sie im Bergwerk jede Staubentwicklung verhindcrt. Die brauchbare Phosphatschicht liegt im Mittel 20-40 m unter der Erdoberfläche und läuft im großen ganzen, von lokalen Abweichungen abgesehen, horizontal dahin. Die Ausbeutung muß also unter Tag erfolgen. Das geschieht unschwer von den wenigen, breiteren Einschnitten aus, die die Flüsse in die einförmige Landoberfläche bis zur Phosphatschicht hinab gegraben haben. Von diesen Tälern gehen an drei Stellen mehrere Kilometer lange Hauptstollen aus, von denen nach beiden Seiten viele Nebenstollen abzweigen. An deren Enden wird das Phosphat ohne besondere Schwierigkeit, bloß mit dem Pickel, abgebaut und dann auf Rollwagen geschaufelt. Diese werden zu kleineren Zügen zusammengestellt, die, an ein elektrisch laufendes Drahtseil gekettet, aus dem Bergwcrk hinausfahren. In kurzen Abständen folgt bei Hochbetrieb, wie er gegenwärtig herrscht, Tag und Nacht ein Züglein dem andern. Über Tag wird der Inhalt der Rollwagen in tiefer stehende Eisenbahnwagen gekippt, die ihn von den drei Abbaustellen

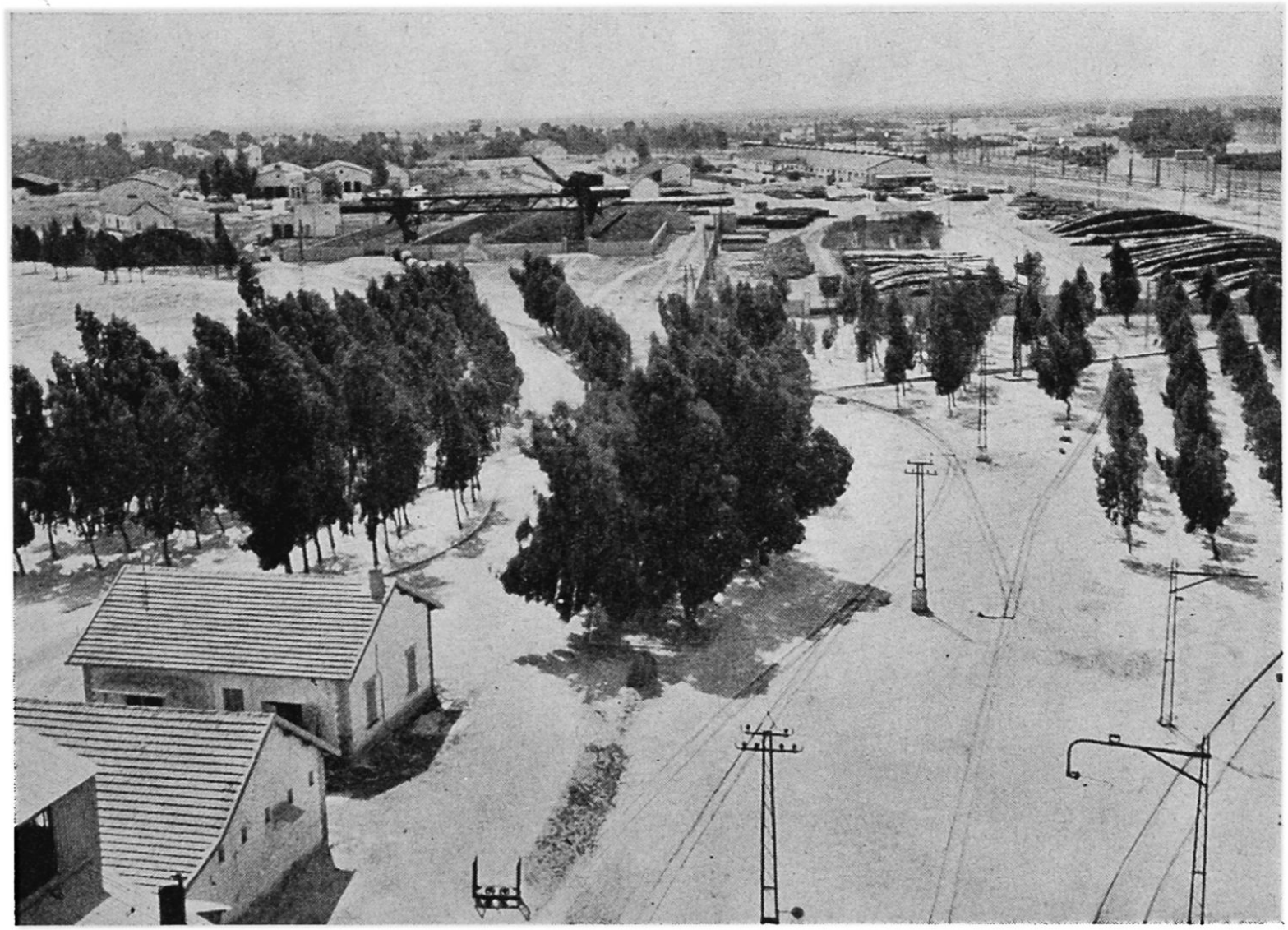

Fig. 4. Khouribga: im Vordergrund ein Teil der Phosphatwerke, im Hintergrund die Europäersiedlung

nach Khouribga führen. Hier finden sich die großen Einrichtungen zur Trocknung und Reinigung der Phosphate. Der Entzug der unbrauchbaren, kalkigen und kieseligen Beimischungen, die im allgemeinen gröber als das Phosphat selber sind, erfolgt durch einfaches Sieben in großen Siebanlagen. Für das Trocknen des weitaus größten Teiles benutzt man eine Anzahl auf Gluthitze erwärmte Öfen, die das Material während 30 Minuten zu passieren hat. Die restliche Menge, ungefähr $150000 \mathrm{t}$ jährlich, wird an Sonne und Luft getrocknet. Das ist aber nur im Sommer während der drei bis vier heißesten Monate möglich. Arbeiter breiten dann auf einem Platze von über 10 ha Fläche eine bestimmte Menge der Phosphate aus und pflügen diese von Zeit zu Zeit um, damit auch tiefere Schichten nach oben an die Luft gelangen. Sie verwenden dazu Metallpflüge, vor die Maultiere, noch häufiger Kamele, gespannt werden. Unter günstigen Umständen ist die ausgebreitete Menge nach sechs Tagen trocken. Täglich fahren mehrere vollbeladene Züge nach dem $100 \mathrm{~km}$ entfernten Hafen von Casablanca.

Es bestehen im ganzen, wie bereits erwähnt, drei Abbaustellen. Zwei davon, die in den Jahren 1923 und 1924 eingerichtet wurden, liegen $4 \mathrm{~km}$ im Südosten von Khouribga, im Tal des Oued Sidi-Bou-Lanouar. Die dritte, die seit 1921 betrieben wird, findet sich in der Senke von Bou Iniba, $16 \mathrm{~km}$ im Osten der Stadt. Jedem Arbeitsplatz 
ist eine verhältnismäßig große Fläche, die ungefähr $10 \mathrm{~km}^{2}$ mißt und eine Phosphatmenge von 15-20 Millionen Tonnen enthält, zur Ausbeutung zugeteilt. Man rechnet für einen solchen mit einer Lebensdauer von ungefähr 30 Jahren. Nur unter dieser Voraussetzung verlohnt es sich, hier die unbedingt notwendigen, aber kostspieligen Einrichtungen zu schaffem, wie Büros, Lagerschuppen, Reparaturwerkstätten, Geleiseanlagen, elektrische Installationen usw.

Noch vor 1921 war das Gebiet von Khouribga äußerst dürftig besiedelt; es war eine fast menschenleere, besonders im Sommer trostlos öde und kahl gebrannte Landschaft. Das änderte mit der Inbetriebnahme der Phosphatwerke mit einem Schlage. Europäer wanderten ins Bled ein und ließen sich als Fachleute, wie Ingenieure, Techniker, Betriebsleiter, Büroangestellte, nieder. Dazu gesellte sich die Schar der als Bergarbeiter angeworbenen Eingeborenen, die aus verschiedenen Teilen Marokkos, vor allem aus dem Großen Atlas vom arbeitsfreudigen Stamme der Schleuh, herkamen. Ende 1945 zählte das Unternehmen 5794 Arbeiter und Angestellte, nämlich 498 Europäer und 5296 Eingeborene. Für alle diese Leute und deren Familien mußte das Phosphatamt für Unterkunft und Verpflegung sorgen. Es schuf deshalb, von 1925 an, die Siedlung Khouribga. Es ließ für die Europäer in einem besonderen Viertel 318 modernere, städtisch aussehende und von kleinen Gärten umgebene Häuser, darunter viele Villen, erbauen. Sie enthalten zusammen 686 neuzeitlich eingerichtete und mit Wasser und Elektrizität versehene Wohnungen. Das Trinkwasser wird aus vielen Kilometern Entfernung hergeleitet und in einem mitten in der Stadt gelegenen Reservoir aufgespeichert. Auch hatte das Phosphatamt verschiedene öffentliche Gebäude zu erstellen, wie Post, Verwaltungsgebäude und Volks- und Berufsschulen; Europäer und Eingeborene sollen beruflich auf den Bergbau vorbereitet werden. Es war ferner notwendig, ein Spital zu errichten, liegt Khouribga doch allzuweit von den wichtigeren marokkanischen Städten entfernt. Der körperlichen und geistigen Förderung der europäischen Einwohnerschaft dienen Sportanlagen verschiedener Art, ein Haus für theatralische, musikalische und kinematographische Vorführungen und ein Klubhaus mit Lese- und Spielsälen, den Ingenieuren reserviert. Der Unterhalt all dieser Gebäude und ferner jener der Straßen, Plätze und öffentlichen Anlagen, kurz der ganzen Europäersiedlung, ist Sache des Phosphatamtes.

Wohltuend für das Auge hebt sich die kleine Stadt mit ihren roten Dächern und grünen Baumgruppen, darunter sogar einem 10 ha großen Eukalyptuswalde, aus der Einöde des Bled heraus. Die Baumbestände sollen in erster Linie den Staub von der Siedlung abhalten, den heftige Winde oft zutragen.

Wenig abseits des Europäerviertels, der Fabrikanlage gegenüber, entstand eine Eingeborenensiedlung. Sie hat das Aussehen einer kleinen Medina und besitzt alles, was zu einer solchen unbedingt gehört, wie Moschee, Koranschule, Backofen, Waschhaus, maurisches Café, maurisches Bad, größerer Platz mit Verkaufsläden, den "Souks", Stadtmauer. Die Häuser sind weiß getüncht, niedrig, von arabischem Stil, gepflegt und sauber; alles ist eben noch verhältnismäßig neu. Vor der Medina dehnt sich ein "douar" aus, ein typisches marokkanisches Eingeborenendorf mit zahlreichen kegelartigen Schilfund Strohhütten, den "noualas", wo sich das Leben in den althergebrachten bäuerlichen Formen abspielt. Die Leute bebauen den Acker; viele Männer arbeiten außerdem noch in den Phosphatwerken. Es ist dies das "village de stabilisation" (Anpassungssiedlung). Hier soll den zugewanderten Eingeborenen, die sich im Bergwerk betätigen wollen, der Übergang von Nomadenleben und bäuerlicher Beschäftigung zu festem Wohnsitz und Lohnarbeit erleichtert werden; man will sie nicht plötzlich und ganz ihren früheren Lebensgewohnheiten entfremden. Darum bekommt hier jeder Arbeiter gratis eine Hektare bewässerbaren Landes zur Bebauung zugeteilt. Im Maße, wie sich die Phosphatwerke entwickelten, wuchs die Zahl der zugewanderten Einwohner, und mit den Arbeitern und Angestellten ließen sich auch Gewerbetreibende, Krämer usw. in Khouribga nieder.

Im Bergwerksbezirk sind in den letzten Jahren für die Eingeborenen noch zwei weitere Siedlungen entstanden: Sidi-Bou-Lanouar bei den Abbaustellen Nummer 1 und 2, und Bou-Iniba bei jener mit Nummer 3. Damit wird für sie der Weg zum Arbeitsplatz, der von Khouribga aus $4-16 \mathrm{~km}$ betragen würde, ganz beträchtlich abgekürzt. Die beiden neuen Orte sehen der Medina von Khouribga auffallend gleich. 


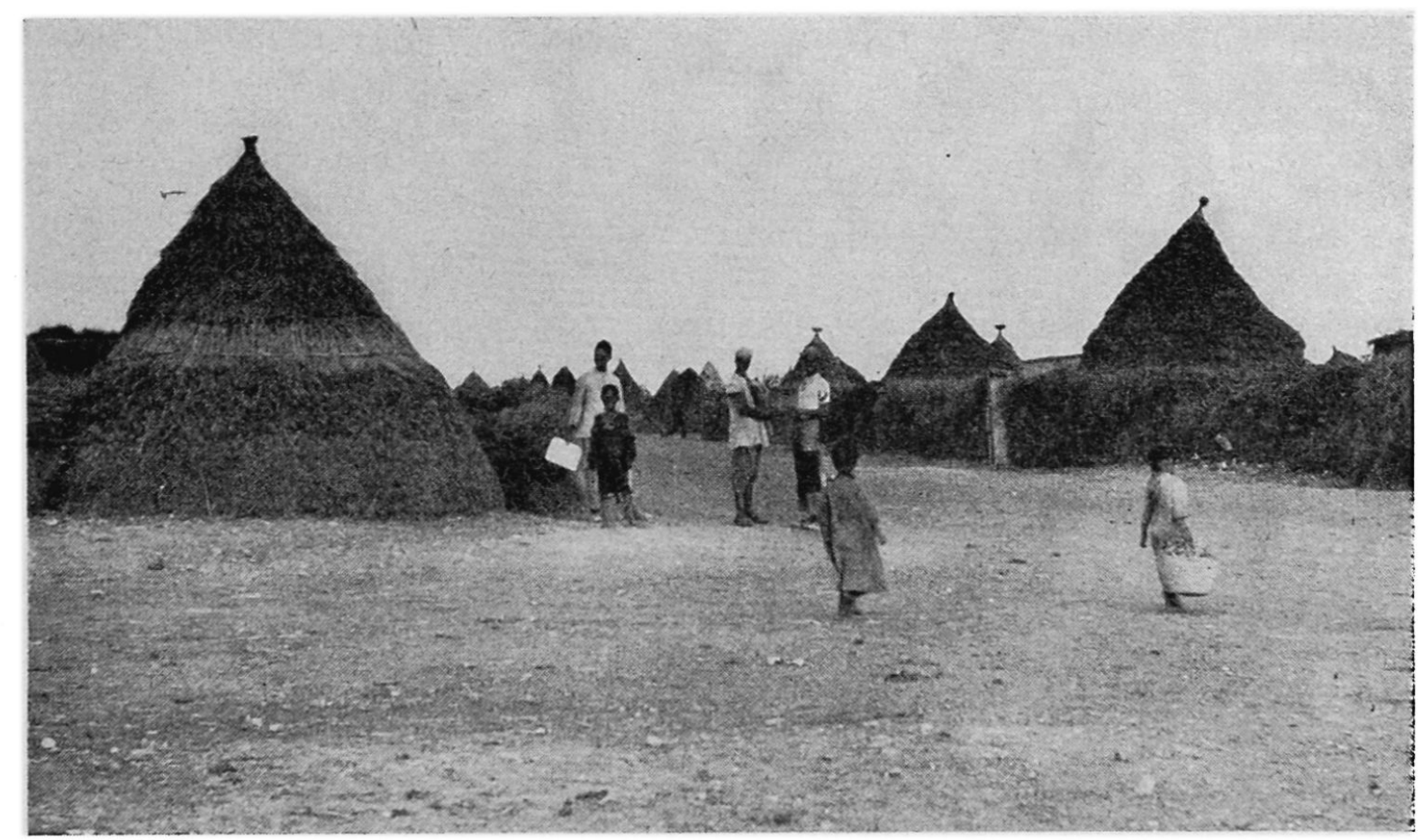

Fig. 5. Khouribga: der aus Schilf- und Strohhütten bestehende «douar» der Eingeborenen (village de stabilisation)

Auch sie gehören dem OCP. Die Zahl der Wohnungen, die das Phosphatamt in diesem Bergbaudistrikt allein für die Eingeborenen bis Ende 1945 erstellt hat, beläuft sich auf 1338. Infolge der Ausbeutung der Phosphate hat sich hier das Aussehen einer Landschaft innerhalb des kurzen Zeitraums von zwei Jahrzehnten grundlegend geändert. Ein vordem fast menschenleerer Raum ist verhältnismäßig dicht bevölkert und besiedelt worden. Er zählt gegenwärtig rund 50000 Einwohner.

Einen etwas anderen Charakter als das nordmarokkanische Lager hat dasjenige von Louis-Gentil im Süden. Wohl erfolgt auch hier der Abbau in durchaus ähnlicher Weise; die Phosphate aber sind mit einem durchschnittlichen Gehalt von $70 \%$ weniger hochwertig. Die Ausbeutung bleibt stark hinter der von Khouribga zurück; sie betrug zum Beispiel 1945 bloß 469000 t. Das Material geht nach dem Hafen Safi. Dort findet sich im Hafen, ähnlich wie in Casablanca, die Einrichtung zum Aufstocken der Phosphate und zum Verladen auf die Schiffe. Das kann in kurzer Zeit geschehen. Laufende Gummibänder befördern das Material von den Zügen oder von den Lagerschuppen direkt aufs Schiff.

Louis-Gentil ist 1935 gegründet worden. Es ist viel kleiner als Khouribga und besteht nur aus 82 vom OCP. für die europäischen Angestellten erbauten Häusern, die zusammen 198 Wohnungen enthalten. Das Werk beschäftigte hier Ende 19451852 Mann, nämlich 180 Europäer und 1672 Eingeborene. Louis-Gentil weist heute im ganzen 4500 Einwohner auf.

\section{HANDWERK UND INDUSTRIE}

Bei der Eingeborenenbevölkerung, insbesondere bei jener, die in den Städten wohnt, ist immer noch das Handwerk von großer Wichtigkeit. Es arbeitet in erster Linie für den Inlandbedarf. Es liefert dem marokkanischen Bauern und Städter eine Menge von Gebrauchsgegenständen, so die aus Wolle gewobenen weißen Überwürfe, die «djellabas ", die Lederpantoffeln, Töpfe, Zeltteppiche und ferner den im Protektorat lebenden 
Europäern eine große Anzahl Luxusartikel, wie Teppiche, Kissen, Metallteller, Seidenwaren. In einigen Städten, zum Beispiel Fes, Marrakesch, Meknes, ist ungefähr ein Drittel der Bevölkerung im Handwerk tätig. Diesem Erwerbszweig drohen jedoch von zwei Seiten große Gefahren: durch die Maschine, die auch bei den Arabern, wenn auch nur langsam, Eingang findet und die Handarbeit allmählich verdrängt, und durch den Einzug der Industrie, die zum Schaden des einheimischen Gewerbes billigere Massenware auf den Markt wirft. Überdies hat die Kriegszeit selber innerhalb dieses Berufsstandes gewisse Veränderungen bewirkt. So sind zum Beispiel jene Körperschaften, die, wie die Keramiker, Vergolder, Messingschläger, die Rohstoffe für ihre Arbeit aus dem Ausland beziehen müssen, schwer mitgenommen worden, weil die Einfuhr ausblieb. Ihre Mitglieder wurden arbeitslos und versuchten, sich begünstigteren Zünften anzuschließen. Von diesen konnten einige wenige, insbesondere jene, die Wolle oder Kupfer verarbeiten, sich während des Krieges vorteilhaft entwickeln, da keine ausländischen Erzeugnisse mehr auf den Markt kamen. Bereits müssen, soll der Handwerkerstand mit all seinen reizvollen Eigenarten voll erhalten bleiben, Maßnahmen zu seinem Schutze getroffen werden. Man will nun zwischen Handwerk und Industrie eine gewisse Teilung der Arbeitsgebiete vornehmen. So soll sich das Handwerk nach wie vor der Herstellung jener Dinge, die für das Land besonders typisch sind, wie Teppiche, Lederwaren, gänzlich widmen.

Marokko ist ein Bauernland. Ackerbau und Viehzucht sichern der Bevölkerung, von der nur ein Sechstel in den Städten wohnt, ihr Dasein. Die Industrie ist noch nicht bedeutend, obwohl sie sich während der zwanzig Jahre ihres Bestehens durchaus bemerkenswert entwickelt hat. Sie vermag einstweilen bloß die dringendsten Bedürfnisse des Landes zu befriedigen, und ihre Ausfuhr beschränkt sich auf einige wenige Erzeugnisse. Es bestehen gegenwärtig, wenn selbst die kleinsten Betriebe mitgezählt werden, 800 industrielle Unternehmen, die zusammen 5000 Europäer und 70000 Eingeborene beschäftigen. Am wichtigsten ist die Lebensmittelindustrie. Zu dieser gehören zahlreiche Getreide- und Ölmühlen, Zuckerraffinerien, die eingeführten Zucker verarbeiten, Konfitüren-, Teigwaren-, Biskuit-, Konservenfabriken und Bierbrauereien.

Eine größere Wichtigkeit hat die industrielle Verwertung der Fische erlangt. Die atlantische Küste Marokkos, ganz besonders die Bucht von Agadir, ist äußerst reich an Fischen, namentlich an Sardinen, aber auch an Thunfisch, Sardellen und Makrelen. Der Fischfang größeren Ausmaßes setzte erst ein, nachdem die Franzosen vom Land Besitz genommen hatten. Die Eingeborenen ließen sich vordem durch die unwirtliche, an guten Häfen arme Küste abhalten. Auch fehlten ihnen die Einrichtungen, die zum Aufbewahren und zum raschen Verfrachten der Fische notwendig sind, besonders unter den gegebenen klimatischen Verhältnissen. Waren 1922 nur ungefähr 500 Einheimische als Fischer tätig, so sind es 1946 ihrer fast 3500. Das macht mit den Europäern zusammen ein Fischervölklein von 4560 Mann. Die Ausbeute ist gewaltig angestiegen, nämlich von $2200 \mathrm{t}$ im Jahre 1922 auf $44000 \mathrm{t}$ im Jahre 1945. Davon entfällt über die Hälfte auf Sardinen. Heute besteht eine eigentliche Fischerflotte von rund tausend größeren und kleineren Schiffen. Ihrer viele sind allerdings zu klein und veraltet und den heutigen Anforderungen der Hochseefischerei nicht mehr gewachsen. Diese Flotte soll in den nächsten Monaten neu ausgestattet und ergänzt werden. Denn Marokko hat erkannt, daß es im unerschöpflichen Fischreichtum seiner Westküste eine wirtschaftliche Möglichkeit allerersten Ranges besitzt.

Der Verbrauch der marokkanischen Bevölkerung an Fischen beziffert sich auf ungefähr 9000 t. Der weitaus größere Teil der Produktion kommt zur Ausfuhr. Der bedeutendste Fischerhafen des Protektorats ist heute Safi. Hier allein sind während der Saison 1945/46 25000 t Sardinen eingebracht und von seinen 20 Konserven- und 25 Einsalzfabriken, die zusammen 5000 Personen beschäftigen, verarbeitet worden. Die 
Fabriken liegen längs der Küste des südlichen Stadtviertels. Welche Bedeutung die Fischverarbeitung für Safi in verhältnismäßig kurzer Zeit erlangt hat, ermißt man aus der Tatsache, daß es im Jahre 1934 nur drei Fabriken besaß. Safi ist im Jahre 1946 sogar zum ersten Sardinenhafen der Welt emporgerückt. Gegenwärtig bestehen in ganz Marokko 129 Unternehmen, die zum Einsalzen und Räuchern, und 50, die zur Konservenherstellung eingerichtet sind. Davon liegen die meisten, von Safi abgesehen, in Casablanca und Fedala. Alle Konservenfabriken zusammen stellten im Jahre 1945 fast eine halbe Million Büchsen her und 1946 rund 800000 .

In Marokko ist dank der seit Jahren anhaltenden regen Bautätigkeit die Zementindustrie wichtig geworden, ferner die Korkindustrie. Der Kork wird nicht mehr wie noch vor wenigen Jahren nur in rohem Zustand ausgeführt, sondern häufig vorher im Lande selber veredelt und dann auf den Weltmarkt gebracht. Die Korkeiche gedeiht in Marokko, namentlich im Walde von Mamora, der im Osten von Rabat liegt, ausgezeichnet. Sie liefert, von ihrer ersten, zwischen ihrem 20. und 30. Lebensjahr fälligen Ernte abgesehen, immer einen Kork von außerordentlicher Güte. Er ist weich, biegsam, sauber und von schöner Farbe und eignet sich bestens zur Herstellung von Zapfen, Korksohlen, Isolierplatten, Tropenhelmen und Korkteppichen. Er ist deshalb sehr gesucht. Seine Produktion nimmt ständig zu, steht aber zurzeit noch weit hinter jener an Kork minderer Qualität zurück (1945 5000 t gegenüber 20000 t). - Auch die chemische Industrie hat Einzug gehalten. Sie fabriziert hauptsächlich Seifen und Kerzen.

Die Industrialisierung Marokkos wird im Maße, wie sich seine Landwirtschaft, sein Bergbau und seine Kraftwerke entwickeln, weiter zunehmen. Bereits bestehen einige, in den letzten zwölf Jahren gebaute Kraftwerke mit bedeutenden Stauanlagen, so zwei am Oum er Rebia, drei am Sebou, eines, das noch nicht voll betriebsfähig ist, bei Imfout usw. In den nächsten drei Jahren wird mit dem Bau weiterer Kraftwerke begonnen werden, so mit jenem am Oued El Abid, für das ein Stausee von $33 \mathrm{~km}^{2}$ Fläche und einem Wasserfassungsvermögen von einer Milliarde Kubikmeter geplant ist. Die Stauwerke sollen nicht nur die Städte und Dörfer, im besondern auch die Industrie, mit Strom versorgen, sondern gleichzeitig noch, was ebenso wichtig ist, zur Bewässerung ausgedehnter Gebiete dienen. Diese Doppelaufgabe ist für die Kraftanlagen Marokkos besonders charakteristisch.

Die Franzosen haben in ihrem Protektorat bereits ein beachtenswertes Werk vollbracht. Dessen wird man sich besonders bewußt, wenn man noch ihre Leistungen auf anderen Gebieten mitberücksichtigt. So hat sich zum Beispiel auch das Verkehrsnetz gewaltig entwickelt. Marokko besitzt seit $1938 \mathrm{mehr}$ als $8100 \mathrm{~km}$ Straßen, worunter fast $5000 \mathrm{~km}$ asphaltierte, breite Hauptstraßen, und mehr als $33000 \mathrm{~km}$ fahrbare Pisten und Militärstraßen. Dazu kommt noch ein Eisenbahnnetz von $1700 \mathrm{~km}$.

\section{LE MAROC D'A PR E S - G U E R RE}

L'étude traite du développement économique du Maroc. Des efforts remarquables sont faits dans le secteur agricole par la fondation d'entreprises collectives, organisées par le "Secteur de modernisation du paysannat ". L'industrie s'intensifie, de même que la pêche (aux sardines avant tout) sur la côte ouest surtout, et les mines de phosphates commencent à prendre de l'importance. La ville de Casablanca, ainsi que d'autres encore, se trouvent en plein essor.

\section{IL MAROCCO NELLA DOPOGUERRA}

Lo studio tratta dello sviluppo economico del Marocco. Sforzi notevoli sono stati fatti nel campo agricolo, sopratutto con la fondazione dell'impresa collettiva organizzata dal "Secteur de modernisation du paysannat ". L'industria è in sviluppo, la pesca (delle sardine) s'intensifica specialmente lungo la costa occidentale. Le miniere di fosfato assumono inoltre un'importanza maggiore. Riguardo alla popolazione e all'ubicazione delle case, Casablanca e altre città sono pure in pieno sviluppo. 